\title{
Comparison of different methods for average anatomical templates creation: do we really gain anything from a diffeomorphic framework?
}

\author{
Vladimir S. FONOV, D. Louis COLLINS \\ McConnell Brain Imaging Centre, Montreal Neurological Institute, McGill University, \\ Montreal, Canada.
}

\begin{abstract}
In the field of computation anatomy, the diffeomorphic framework is widely used to perform analysis of human brain anatomy in both healthy and diseased populations. While useful for analysis, the framework imposes certain implementation constraints that do not necessarily result in improved accuracy of inter-subject co-registration in case of average anatomical template (AAT) construction - a common technique used in large population studies. In this work, we evaluated several state-of-the-art non-diffeomorphic and diffeomorphic non-linear registration frameworks in terms of their ability to build AATs. While all methods generated well behaved transforms, we found that the diffeomorphic framework does not automatically guarantee an increase of accuracy in average anatomical template construction.
\end{abstract}

Keywords: Non-linear registration, diffeomorphism, average anatomical template

\section{Introduction}

Average anatomical templates (AAT) are useful tools in computational anatomy (Mazziotta, Toga et al. 1995). They are applied in a wide variety of methods requiring registration to a common space for comparison, for example when performing VoxelBased Morphometry (VBM) analysis, serving as priors for the automatic tissue classification and automatic anatomical structure segmentation. Multiple strategies for creating AATs have been created during the last 19 years. Starting with voxel-wise arithmetic averaging of manually registered scans (Evans, Collins et al. 1993), automatic linear coregistration (Collins, Neelin et al. 1994) and then non-linear average templates using elastic registration (Guimond, Meunier et al. 1998), free-form deformation with B-Splines (Bhatia, Aljabar et al. 2007), and large deformations diffeomorphic mapping (LDDM) (Lorenzen, Davis et al. 2005). Several studies testing the impact of template construction have been published (Wang, Seghers et al. 2005), (Klein, Ghosh et al. 2010), (Avants, Tustison et al. 2011) with overall conclusion that templates based on the average shape and intensity perform better in anatomical structure segmentation then direct pair-wise registration.

Among the various currently available methods, one important distinction appears: some methods are based on a diffeomorphic framework (Avants, Tustison et al. 2011), (Lorenzen, Davis et al. 2005), while others aren't (Guimond, Meunier et al. 1998), (Bhatia, Aljabar et al. 2007). The diffeomorphic template building framework guarantees that the generated transforms are smooth, invertible, bijective maps between all volumes, such that the forward and inverse transforms are differentiable. This preserves object topology through the transform, and allows using methods developed in the field of differ- 
ential geometry and generally facilitates analysis. While in theory the use of a linear elastic regularizer (e.g., (Collins, Holmes et al. 1995)) or arithmetic operations on vector deformation fields do not guarantee a diffeomorphic transformation, in practice this is often the case because of the constraints implemented in optimization and regularization; the resulting transformations are smooth, invertible and both forward and inverse transforms are differentiable numerically. Some of these constraints are not unlike those needed for numerical implementation of a diffeomorphic mapping on a discrete grid. While some researchers view the diffeomorphic framework as a kind of silver bullet, able to automatically improve and/or justify results of statistical morphological studies of human brain anatomy, how do these imposed constraints affect average anatomical template construction? Are diffeomorphic registration methods really needed?

The purpose of this study is to evaluate if the diffeomorphic framework guarantees better results in the limited scope of building average anatomical templates, where better is defined in the sense of improving co-registration accuracy between subjects chosen to create the template. In this paper, five algorithms based on the publicly available tools are compared, with the ANTS method (Avants, Tustison et al. 2011) and simple linear average serving as a baseline reference. In the spirit of reproducible research, the source code for these comparisons is made publicly available for at the url: http://anonymous.web.site [note: anonymous only during review].

\section{Materials and Methods}

In this study, two databases are used. First, T1-weighted (T1w) MRI data from 152 normal subjects from the International Consortium for Brain Mapping (ICBM) project $\left(1 \mathrm{~mm}\right.$ thick slices, $\mathrm{TR}=18 \mathrm{~ms}, \mathrm{TE}=10 \mathrm{~ms}$, flip angle $30^{\circ}$ with $1 \mathrm{~mm}$ in-plane resolution, acquired on a 1.5T Philips Gyroscan scanner (Mazziotta, Toga et al. 1995)); and second, MRI scans and manually segmented labels of 40 normal subjects from Probabilistic Brain Atlas (LPBA40) at the Laboratory of Neuro Imaging (LONI) at UCLA (Shattuck, Mirza et al. 2008) (High-resolution 3D Spoiled Gradient Echo (SPGR),1.5-mm slices, TR range 10.0-12.5 ms; TE range 4.22-4.5 ms; in-plane voxel resolution of $0.86 \mathrm{~mm}$ or $0.78 \mathrm{~mm}$, acquired on a GE $1.5 \mathrm{~T}$ scanner).

Anatomical scans from ICBM project were corrected for image intensity inhomogenieties (Sled, Zijdenbos et al. 1998), linearly registered to MNI152 stereotaxic space (Collins, Neelin et al. 1994), the intensity range was linearly normalized to $[0,100]$ using histogram matching and brain extraction was performed using BET (Smith 2002). Scans in "delineation space" from the LPBA40 database were already processed for intensity inhomogeneity and stereotaxic registration, and were only normalized to be in the $[0,100]$ interval and used together with the manually-defined anatomical labels describing 55 structures.

Scans from both databases were automatically segmented into three brain tissue classes: white matter (WM), gray matter (GM) and cerebro-spinal fluid (CSF) using unsupervised finite mixture model classifier (Tohka, Krestyannikov et al. 2007) which does not require usage of spatial priors. These tissue labels (and the anatomical labels of the LPBA40) are used below in the goodness of fit metrics used to compare the different registration strategies. 


\section{Minimum deformation template}

The algorithm to build a minimum deformation template is similar to that described in (Avants, Tustison et al. 2011) (in the script buildtemplateparallel.sh available in ANTs source code) and in (Fonov, Evans et al. 2011). The strategy used here is formulated as the following:

1. For a given template $T_{i-1}$ and subjects $I_{k}$ (where $k=1 . . K$ ), find (non-linear) transformations $S_{i, k}$ minimizing $\boldsymbol{D}\left(T_{i-1}, I_{k}\left(S_{i, k}\right)\right)$ for each subject $k$, where $\boldsymbol{D}$ represents the costfunction used, optionally using $F_{i-1, k}$, the non-linear transformation from the previous iteration for initialization.

2. Estimate the Frechet mean, $Z_{i}$, in the space of transformations, minimizing $\sum_{k} \boldsymbol{\Delta}\left(Z_{i}, S^{-1}{ }_{i k}\right)$, where $\Delta$ represents a distance function in the space of non-linear transformations, and $S$ ${ }_{i k}$ is the inverse of $S_{i k}$, such as $S^{-1}{ }_{i k} \circ S_{i k}=I d$.

3. Calculate $F_{i k}=\boldsymbol{U}\left(S_{i k}, Z_{i}\right)$, where $\boldsymbol{U}$ is the update rule.

4. Estimate new template $T_{i}$, minimizing $\sum_{k} \boldsymbol{D}\left(T_{i}, I_{k}\left(F_{i k}\right)\right)$. In the case when $\mathrm{D}$ is crosscorrelation or sum of squared differences, the arithmetic mean is the solution: $T_{i}=1 / K \cdot \sum_{k} I_{k}\left(F_{i k}\right)$,

5. Repeat steps 1-4 until convergence

It is possible to adopt above algorithm to use different non-liner registration schemes, both Diffeomorphic and Elastic, and in the current study we have evaluated performance of the following template building methods:

- ANIMAL (Collins, Holmes et al. 1995) elastic non-linear registration algorithm with correlation-coefficient intensity cost function $\boldsymbol{D}$, and sum of squared differences distance $\Delta$, resulting in the $\mathrm{Zi}=1 / \mathrm{K} \cdot \sum_{k} S_{i k}$, and update rule $U(x, y)=x \circ y$.

- Diffeomorphic Demons (DD) (Vercauteren, Pennec et al. 2009) with symmetrized gradients used to compute the demons forces, diffeomorphic non-linear registration algorithm, sum of squared differences cost function $\boldsymbol{D}$, sum of squared differences distance $\Delta$, and update rule $U(x, y)=x \circ y$. Implemented in ITK 3.20.1 (Ibanez 2005).

- Thirion's Demons or Non-Diffeomorphic Demons (NDD), with symmetrized gradients (Vercauteren, Pennec et al. 2009), sum of squared differences cost function $\boldsymbol{D}$, sum of squared differences distance $\boldsymbol{\Delta}$, and update rule $U(x, y)=x \circ y$. Implemented in ITK 3.20.1 (Ibanez 2005).

- Log-space Diffeomorphic Demons (LDD) (Vercauteren, Pennec et al. 2008) with symmetrized gradients, in this case all operations on the transformations are performed in log-domain on velocity fields $V_{i k}$, which can be converted into a diffeomorphic transformation via exponential mapping, i.e $S_{i k}=\exp \left(V_{i k}\right)$. In this case, we use sum of squared differences cost function $\boldsymbol{D}$, sum of squared differences distance $\boldsymbol{\Delta}$ calculated in $\log$ domain and update rule $\mathrm{U}(\mathrm{x}, \mathrm{y})=\mathrm{x}+\mathrm{y}$ also calculated in log-domain.

- Symmetric group-wise normalization template building algorithm (Avants, Tustison et al. 2011) as defined in script buildtemplateparallel.sh in the ANTs package (ANTs) with the exception of disabling intensity normalization and sharpening (LaplaciansharpeningImagefilter) filter inside AverageImages tool, using cross-correlation intensity cost-function.

- Straight linear averaging (LINEAR), i.e., without any non-linear registration. 


\section{Hierarchical processing}

Almost all the template building algorithms described above (apart from LINEAR) use a hierarchical approach for varying parameters of non-linear registration, starting with a rough estimation of deformation (or velocity) fields and progressively increasing resolution. We have adopted a scheme compatible with the one published in (Fonov, Evans et al. 2011): for the ANIMAL method: 4 iterations at $32 \mathrm{~mm}$ resolution of deformation field, 4 at $16 \mathrm{~mm}, 4$ at $8 \mathrm{~mm}, 4$ at $4 \mathrm{~mm}$ and 4 at $2 \mathrm{~mm}$ with 20 iterations in total; for DD,NDD and LDD we have started at 4 iterations at $16 \mathrm{~mm}$ resolution, 4 at $8 \mathrm{~mm}, 4$ at $4 \mathrm{~mm}, 4$ at $2 \mathrm{~mm}$ and 4 at $1 \mathrm{~mm}$; for ANTs we have used the scheme published in (Avants, Tustison et al. 2011) - 3 iterations with $30 \times 50 \times 0$ registrations steps and 3 iterations with $50 \times 90 \times 20$ registration steps. All template building algorithms evaluated here produce a dense deformation field for each subject, enabling it to be warped into the space of the final AAT. These deformation fields may be applied to the tissue classification maps and anatomical labels maps to produce probabilistic atlases of corresponding tissue types and anatomical structures.

\section{Performance metrics}

The relative performance of each algorithm is evaluated using the following metrics:

- Generalized Tannimoto Coefficient (GTC, (Crum, Camara et al. 2006)). GTC is applied to the tissue and anatomical label maps resampled using nearest-neighbour interpolation using the final non-linear deformation fields obtained during template construction. Described by Eq.1, which is generalization of Tannimoto or Jaccard overlap coefficient in the case of fuzzy labels $\mathrm{A}_{\mathrm{kli}}, \mathrm{B}_{\mathrm{kli}}$, multiple labels and multiple subjects.

$$
G T C=\frac{\sum_{\text {pairs }, k} \beta_{k} \sum_{l a b e l s, l} \alpha_{l} \sum_{\text {voxels }, i} \operatorname{MIN}\left(A_{k l i}, B_{k l i}\right)}{\sum_{\text {pairs }, k} \beta_{k} \sum_{l a b e l s, l} \alpha_{l} \sum_{\text {voxels }, i} \operatorname{MAX}\left(A_{k l i}, B_{k l i}\right)}
$$

By collapsing summations depending on the situation, it is possible to apply this formula in different cases (e.g., voxel-wise across all subjects, region-wise between only two subjects, etc...) To evaluate the accuracy of each AAT building algorithm, the GTC was applied on both a voxel-wise level for the tissue-classification and anatomical structures maps warped into the common space, and overall for the whole brain. Also, we have calculated both overall GTC for all subjects participating in AAT building and for all possible pairs to estimate the range of overlap values.

- Harmonic Energy (HE, (Vercauteren, Pennec et al. 2009)). HE is applied to the individual deformation fields, described in Eq. 2 , where $\mathrm{J}$ is Jacobian matrix, $\mathrm{V}$ is the total number of voxels and $\|\cdot\|$ is Frobenius norm. The HE represents the degree of smoothness of the transform. For equivalent GTC, smaller HE is better.

$$
H E=\frac{\sum\|J\|^{2}}{V}
$$

- Sharpness (Sh). Sh is applied to the result of template building process, determined by Eq. 3 where $\mathrm{G}$ is Gaussian smoothing kernel with a full-width half-max (FWHM) of $1 \mathrm{~mm}$. Sh represents relative visual sharpness of the image $I$.

$$
S h=\operatorname{Median}(|\nabla \mathrm{G}(I)|)
$$




\section{Parameter optimization}

Demons-based algorithms have two tunable parameters: deformation field smoothing sigma $s$ and update field smoothing sigma $g$, to control the degree of regularization in the non-linear registration process. The ICBM152 dataset was used to find the optimal values of $s$ and $g$ for the LDD template building algorithm.

\section{Results}

\section{Parameter optimization using ICBM 152 dataset.}

Results of the AAT building algorithm applied to ICBM 152 dataset is shown of Fig. 1. Comparing the GTC overlap ratios and sharpness for LDD, $s=1$ and $g=1$ were chosen as they produced the best results (Fig. 1a). The same parameters were used to create AAT using the DD algorithm. NDD, LDD and ANTs yield the best GTC overlap. Surprisingly, ANIMAL achieves the highest Sharpness results, followed by ANTs and LDD (Fig 1b). The ATT voxel-by-voxel image intensity standard deviation (SD) can also give an idea of registration quality, where lower SD indicates more consistent registrations. NDD, LDD and DD give the best results for SD (Fig 1c).

Figure 2 shows one slice of the AATs produced by each algorithm together with voxelwise GTC overlap ratio map (where higher/brighter voxels indicate better fits between subjects). One can notice that the bulk of the white matter is very well aligned, even when using linear co-registration, however cortex and deep-gray matter structures clearly benefit from using non-linear registration. Also it is worth noting that the cortical folding pattern is slightly different between different methods, whereas deep structures appear very similar.
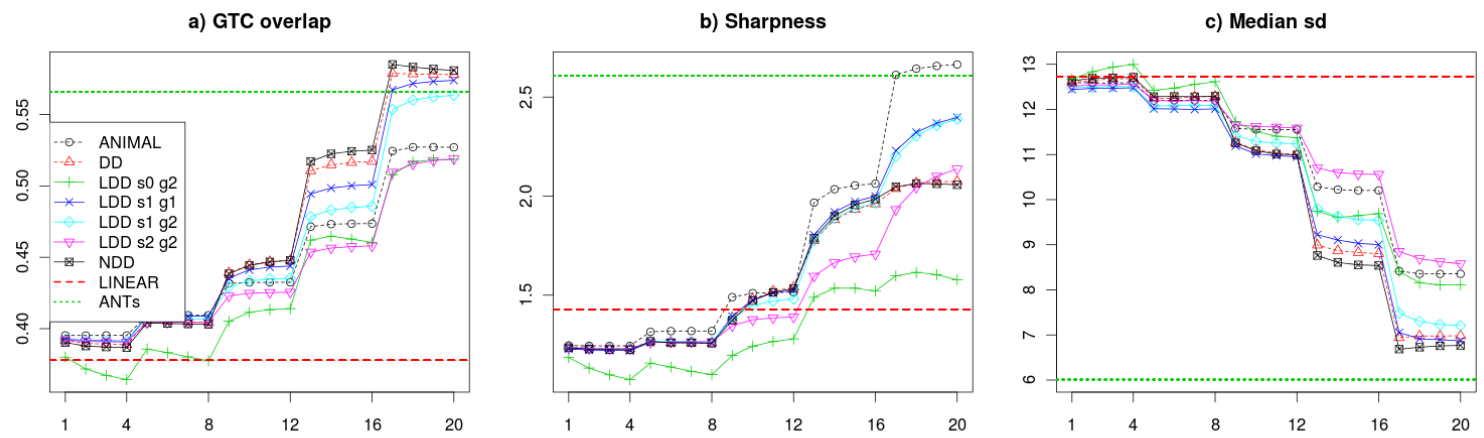

Fig. 1. GTC, sharpness and median standard deviation calculated for each iteration of AAT building using the ICBM 152 dataset 


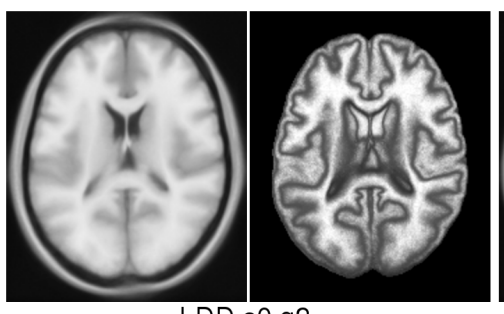

LDD s0 g2
0.518

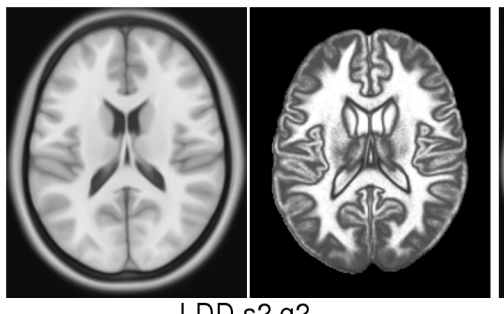

LDD s2 g2

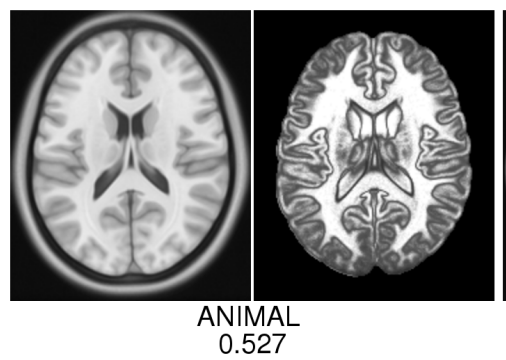

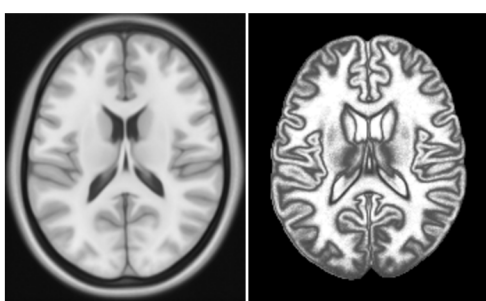

LDD s1 g1
0.573

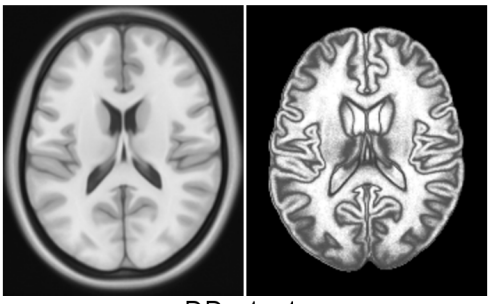

DD s1 g1

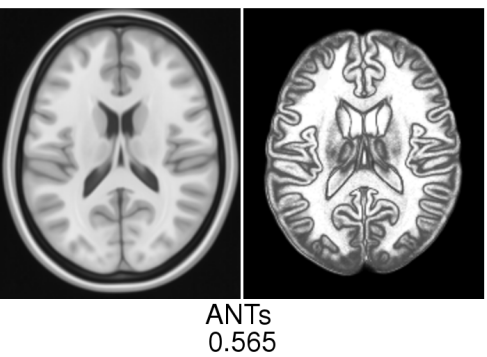

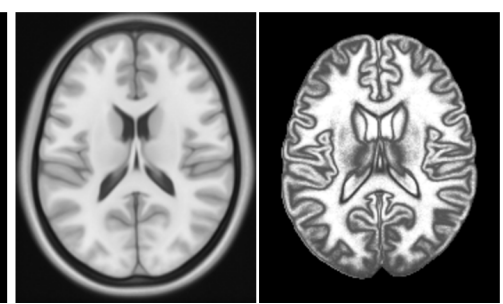

LDD s1 g2
0.563

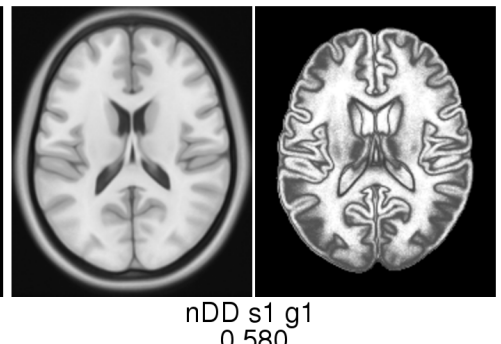

0.580

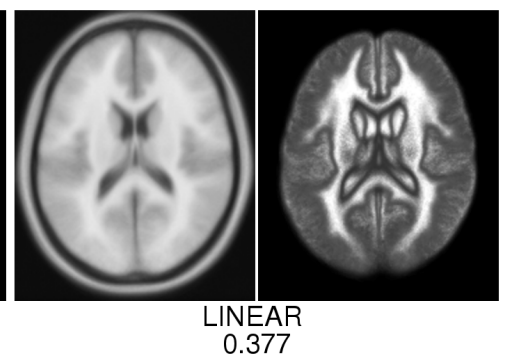

Fig. 2. Final results with ICBM 152 dataset. In each pair, the AAT produced by each algorithm (left) is shown with the voxel-wise GTC map (right), where black corresponds to zero overlap and white, $100 \%$ overlap. The number corresponds to the overall GTC overlap.

\section{Results of LPBA40 Average Anatomical Template construction.}

Figure 3 shows the results of each algorithm on LPBA40 dataset. In contrast to ICBM152 dataset, we have calculated pair-wise GTC overlap ratio between structure label-sets of each subject warped into the space of the AAT, with a total of 780 pairs in each case. The pair-wise tissue GTC results in Fig. 3a show that the three best techniques (in order) are NDD, DD and LDD ( $<<0.00001$, non-parametric Friedman rank sum test). The pair-wise structure GTC results in Fig. $3 b$ show the three best techniques are ANIMAL, NDD and LDD, and while significant, the differences are very small. ANIMAL and ANTs obtain the lowest HE values. Interestingly, while ANTs, ANIMAL and NDD have similar pair-wise structure GTC, the ANTs and ANIMAL HE values are much smaller than NDD, indicating that smoother transforms can achieve similar structure segmentations.

Figure 4 shows the final AAT and GTC overlap maps produced by each method. Note that LPBA40 dataset contains 55 anatomical structures, but we used only 53; cerebellum and brainstem were excluded since these structures were masked away in the delineation space. Figure 4 demonstrates that the overlap ratio is relatively high at the center of each cortical structure, while the edges do not agree very well for all presented algorithms. This reduction in the overlap metric at structure borders might be caused by both anatomical variability of the human cortex and difficulty of delineating anatomical structures by the human raters. 

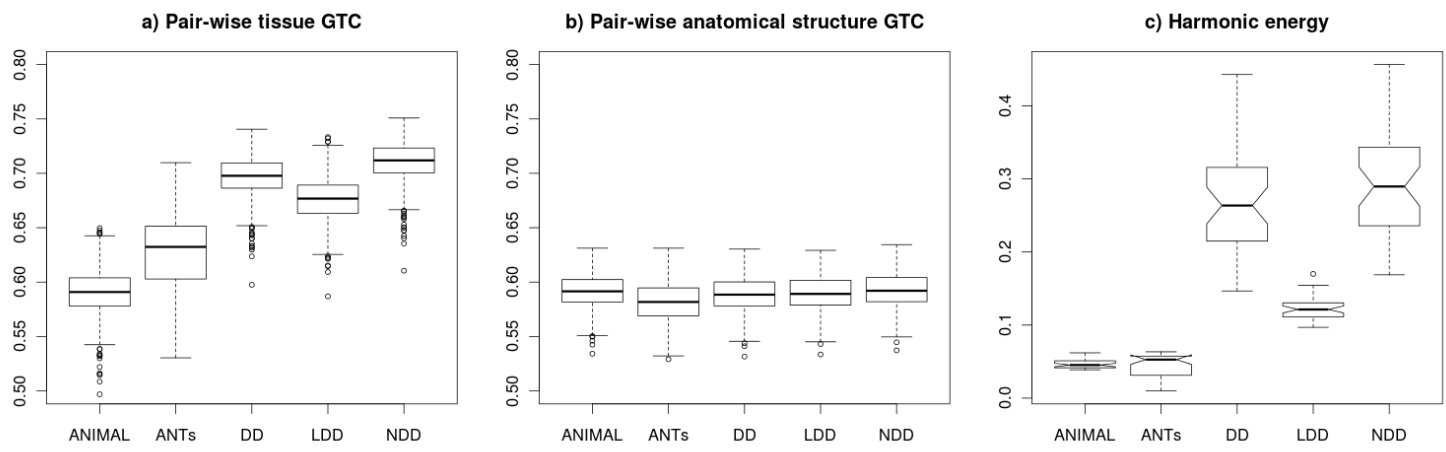

Fig. 3. Pair-wise GTC overlap ratio of tissue classes (a), pair-wise overlap ratio of anatomical structures (b) and $\mathrm{HE}$ distribution of the deformation fields (c) mapping each subject into the algorithm's AAT template space.
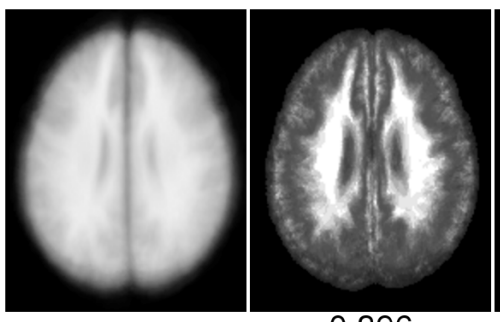

0.396

LINEAR
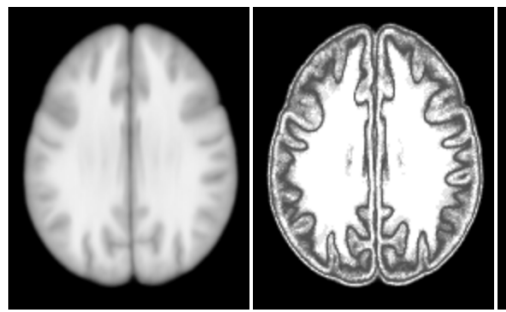

0.625

ANTs
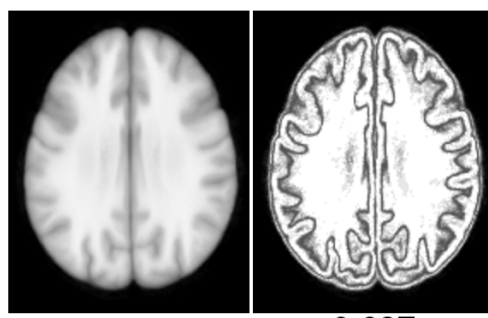

0.687

NDD

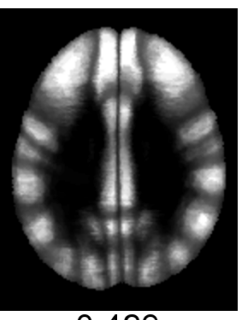

0.429

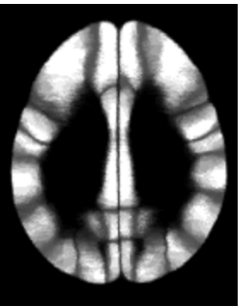

0.581

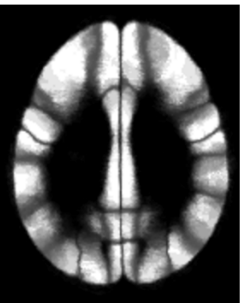

0.570
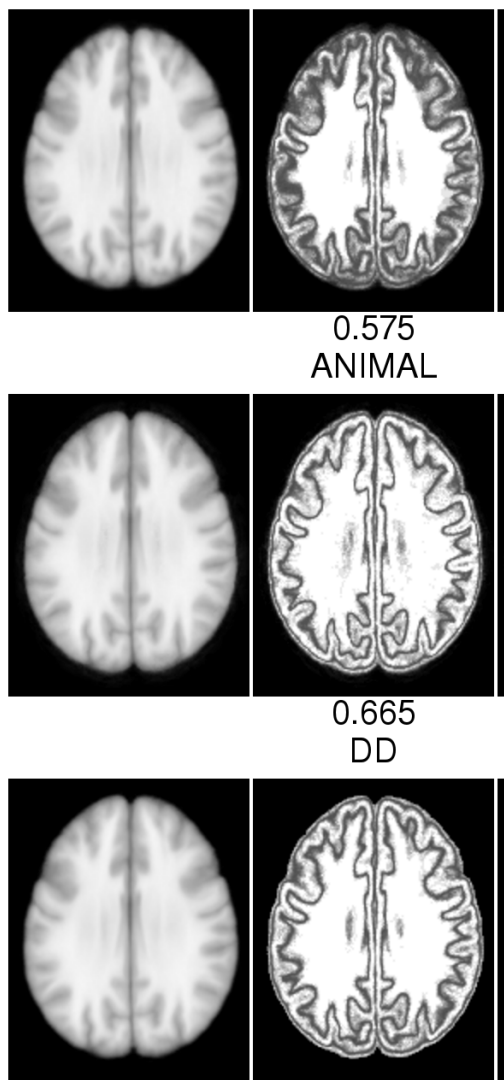

LDD
0.676

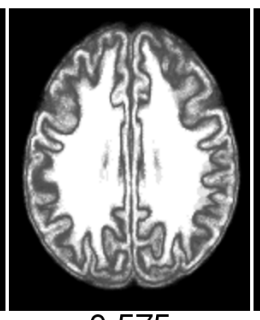

0.575

ANIMAL

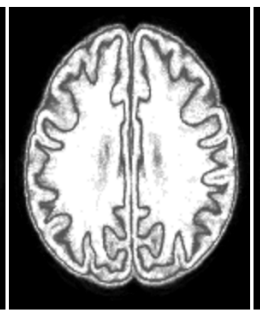

0.665

DD

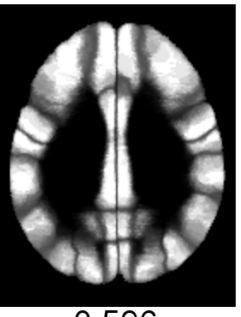

0.596

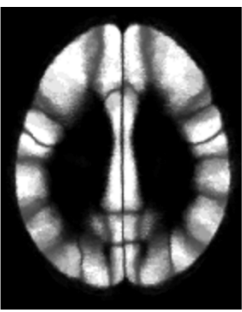

0.557

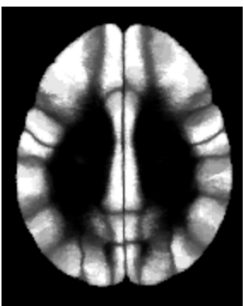

0.589

Fig. 4. AAT and GTC maps of tissue classes and anatomical structures for each method.

\section{Discussion and Conclusions}

Our experiments demonstrate that the NDD, DD and LDD gave the best tissue-class GTC on both the ICBM152 and LPBA40 databases; ANIMAL achieved the highest Sharpness results, followed by LDD. There is very small difference between algorithms w.r.t. pairwise anatomical structure GTC, while ANIMAL and ANTs yielded the smoothest transforms w.r.t. HE. Our experiments show that application of fully diffeomorphic 
methods do not automatically guarantee an increase of accuracy in average anatomical template construction, at least w.r.t overlap of tissue classes and anatomical labels, while all techniques yielded well-behaved transformations. In fact, the overall best performing method in our experiments was Thirion's Demons based algorithm (NDD) with symmetrized gradient calculation (Vercauteren, Pennec et al. 2009).

\section{$5 \quad$ References}

Avants, B. B., N. J. Tustison, G. Song, P. A. Cook, A. Klein and J. C. Gee (2011). "A reproducible evaluation of ANTs similarity metric performance in brain image registration." Neuroimage 54(3): 2033-2044.

Bhatia, K., P. Aljabar, J. Boardman, L. Srinivasan, M. Murgasova, S. Counsell, M. Rutherford, J. Hajnal, A. Edwards and D. Rueckert (2007). Groupwise Combined Segmentation and Registration for Atlas Construction. Medical Image Computing and Computer-Assisted Intervention - MICCAI 2007: 532-540.

Collins, D. L., C. J. Holmes, T. M. Peters and A. C. Evans (1995). "Automatic 3-D modelbased neuroanatomical segmentation." Hum Brain Mapp 3(3): 190-208.

Collins, D. L., P. Neelin, T. M. Peters and A. C. Evans (1994). "Automatic 3D intersubject registration of MR volumetric data in standardized Talairach space." Journal of Computer Assisted Tomography 18(2): 192-205.

Crum, W. R., O. Camara and D. L. G. Hill (2006). "Generalized Overlap Measures for Evaluation and Validation in Medical Image Analysis." Medical Imaging, IEEE Transactions on 25(11): 1451-1461.

Evans, A. C., D. L. Collins, S. R. Mills, E. D. Brown, R. L. Kelly and T. M. Peters (1993). 3D statistical neuroanatomical models from 305 MRI volumes. Nuclear Science Symposium and Medical Imaging Conference, 1993., 1993 IEEE Conference Record.

Fonov, V., A. C. Evans, K. Botteron, C. R. Almli, R. C. McKinstry and D. L. Collins (2011). "Unbiased average age-appropriate atlases for pediatric studies." Neuroimage 54(1): 313-327.

Guimond, A., J. Meunier and J.-P. Thirion (1998). Automatic Computation of Average Brain Models. Medical Image Computing and Computer-Assisted Interventation MICCAI'98: 631.

Ibanez, L. a. S., W. and Ng, L. and Cates, J. (2005). The ITK Software Guide, Kitware, Inc. .

Klein, A., S. S. Ghosh, B. Avants, B. T. T. Yeo, B. Fischl, B. Ardekani, J. C. Gee, J. J. Mann and R. V. Parsey (2010). "Evaluation of volume-based and surface-based brain image registration methods." Neuroimage 51(1): 214-220.

Lorenzen, P., B. Davis and S. Joshi (2005). "Unbiased atlas formation via large deformations metric mapping." Med Image Comput Comput Assist Interv Int Conf Med Image Comput Comput Assist Interv 8(Pt 2): 411-418.

Mazziotta, J. C., A. W. Toga, A. Evans, P. Fox and J. Lancaster (1995). "A Probabilistic Atlas of the Human Brain: Theory and Rationale for Its Development: The International Consortium for Brain Mapping (ICBM)." Neuroimage 2(2, Part A): 89-101.

Shattuck, D. W., M. Mirza, V. Adisetiyo, C. Hojatkashani, G. Salamon, K. L. Narr, R. A. Poldrack, R. M. Bilder and A. W. Toga (2008). "Construction of a 3D probabilistic atlas of human cortical structures." Neuroimage 39(3): 1064-1080. 
Sled, J. G., A. P. Zijdenbos and A. C. Evans (1998). "A nonparametric method for automatic correction of intensity nonuniformity in MRI data." Medical Imaging, IEEE Transactions on 17(1): 87-97.

Smith, S. M. (2002). "Fast robust automated brain extraction." Hum Brain Mapp 17(3): 143-155.

Tohka, J., E. Krestyannikov, I. D. Dinov, A. M. Graham, D. W. Shattuck, U. Ruotsalainen and A. W. Toga (2007). "Genetic Algorithms for Finite Mixture Model Based Voxel Classification in Neuroimaging." Medical Imaging, IEEE Transactions on 26(5): 696-711. Vercauteren, T., X. Pennec, A. Perchant and N. Ayache (2008). Symmetric Log-Domain Diffeomorphic Registration: A Demons-Based Approach

Medical Image Computing and Computer-Assisted Intervention - MICCAI 2008. D. Metaxas, L. Axel, G. Fichtinger and G. Székely, Springer Berlin / Heidelberg. 5241: 754761.

Vercauteren, T., X. Pennec, A. Perchant and N. Ayache (2009). "Diffeomorphic demons: Efficient non-parametric image registration." Neuroimage 45(1, Supplement 1): S61-S72. Wang, Q., D. Seghers, E. D’Agostino, F. Maes, D. Vandermeulen, P. Suetens and A. Hammers (2005). Construction and Validation of Mean Shape Atlas Templates for AtlasBased Brain Image Segmentation. Information Processing in Medical Imaging: 689-700. 